\title{
Muscle Damage and Recovery During a Basketball Competition
}

\author{
Tahir Kilic ${ }^{1 *}$ and Ali Emre Erol ${ }^{2}$ \\ ${ }^{1}$ Department of School of Physical Education and Sports, Akdeniz University, Turkey \\ ${ }^{2}$ Department of School of Physical Education, Gazi University, Turkey
}

Submission: August 29, 2018; Published: September 04, 2018

*Corresponding author: Tahir Kilic, Department of School of Physical Education, Akdeniz University, Turkey, Email: Turkey.tkilic@akdeniz.edu.tr

\begin{abstract}
Muscle damage is characterized by increased serum enzyme levels at blood serum level. The present study aimed to investigate the biochemical variability of the muscle damage indicators at blood serum level such as creatine kinase (CK), lactate dehydrogenase (LDH) and myoglobin (MYB) of the players who had played on four successive days and been subjected to recovery protocol after the contest. 20 healthy men basketball players (/N 20:A $10+\mathrm{B} 10$ ) whose average age is A: $23,70 \pm 2,80 ; \mathrm{B}: 22,80 \pm 2,94$; average height is A: $181,30 \pm 8,20$; $\mathrm{B}: 178,95$ $\pm 4,68$; weight is A: $76,18 \pm 11,31$; B: $69,96 \pm 7,68$, exercise age is A: $11,00 \pm 3,97 ; B: 8,20 \pm 3,55$ have been included in the study.Blood samples were taken on 4-day contest period and 3-day recovery phase that is 24-48-72hours following the contests. The subjects in Group A were applied 10 -minute recovery protocol after the contest.

In this study, considerable increase has been observed in CK, LDH and MYB values in pre-contest and post-contest measurements. MYB and CK values couldn't reach the peak point in the other contests that they had reached after the first contest. While CK values were not influenced by recovery, examining the speed and values of returning to the start level, it was noticed that it was effective on MYB and LDH values. In the analysis of recovery protocol, 10minute recovery protocol wasn't found to be effective on MYB and CK values, but a significant effect was observed on LDH values measured 48-72hours later. In respect to our study, in which muscle damage responses, in accordance with the postcontest measurements, have reached to normal level in a shorter time in the group subjected to recovery protocol after the contest, it could be said that at the end of the basketball tournament there are muscle damage responses in players' skeletal muscles and these responses come to normal level 24-48-72hours later.
\end{abstract}

Keywords: Creatine kinase; Lactate dehydrogenase; Myoglobin; Recovery

\section{Introduction}

Basketball match can be thought as eccentric and slowspeed exercise when compared to high-speed ones in current muscle damage studies. The adaptation to eccentric exercise can be achieved without damaging the muscles [1].Recovery and reversible muscle damage effects, compression garments, cold-water dipping method, ice application, massage after match, ergogenic aids and peak performance are important components of the post-play activities today. Within the domain of recovery strategies, there is little knowledge relevant to studies on minimizing the muscle damage effects or clearing the present waste products.In literature researches, it is observed that intensively loaded eccentric loading protocols that enhance muscle damage are applied to most of the muscle damage recovery exercises[2].

There are a limited number of studies about post-match recovery stage $[3,4]$.Recovery after exercise can be in form of absolute recreation or active recovery.Gentle exercises during the recovery phase after heavy exercises like jogging causes lactic acid such as (35-40 \% Max Vo2) to be removed more quickly. This type of recovery is called recovery with exercise or active recovery. Cool-down exercises after the actual exercise is an example to that and have great contributions to removing the lactic acid [3].

Every sportsman is exposed to oxygen debt when he plays at maximal capacity during the contest and creates some amount of lactic acid. It is necessary to apply recovery protocol for the loadings during the contest or at least 10-minute Cool-down exercise in terms of restoring for the next trainings and contests. Cool-down after a high-speed exercise includes the movements the intensity of which decrease gradually to revert the blood circulation and body functions to the condition before the exercise. The restoration process soon after the load stimulation is an intensive one and it can be generally said that two third of restoration is completed within the corresponding time in which one third of recreation is completed[5].The present study, 
in view of these considerations, has been planned to investigate the effects of the basketball contests on successive days and the repeated movements in such intensive contests on muscle damage and recovery phase.

\section{Methods}

After ethics committee approval,(Clinical Research with Turkish Republic Ministry of Health General Directorate of Medicine and Pharmacy No. and B-10-0-IEG-0-15-00-01 Issue complies with the decision of Non-Pharmacological Clinical Researches dated 17thSeptember 2010). Twenty healthy male volunteer basketball players attended in this study and divided into two groups as Group 1(A) and Group 2(B).The research was designed to last for 7 days in 2 sections.1st section is the tournament that includes 4 contests after and before which the values are measured. (1st, $2 \mathrm{nd}, 3 \mathrm{rd}, 4$ th days). 2 nd section is the recovery that consists of measuring the values in 24-48-72hours after the contests. (5th, 6 th, 7 th days).

The subjects involved in the research were informed not to take any drug one week before the research, not to use alcohol, not to do high-speed exercise except trainings, to inform any injuries and bleedings as these probable conditions may have effects on the analysis results. Twenty healthy male volunteer basketball players attended in this study and divided into two groups as Group 1(A) and Group 2(B).The first subject group was put to 10-minute recovery protocol after the contest. Recovery protocol included jog-trot and stretching exercises. Active rest is; 1-2minute active breathing, 30-60 seconds jogging, 3-5 minutes walking and finally stretching. Inorder to identify the variables in the hematological values of the subjects, venous blood samples were taken from the forearms 11 times. The blood samples were placed in two separate $10-20 \mathrm{ml}$ tubes with EDTA and immediately delivered to the laboratory.

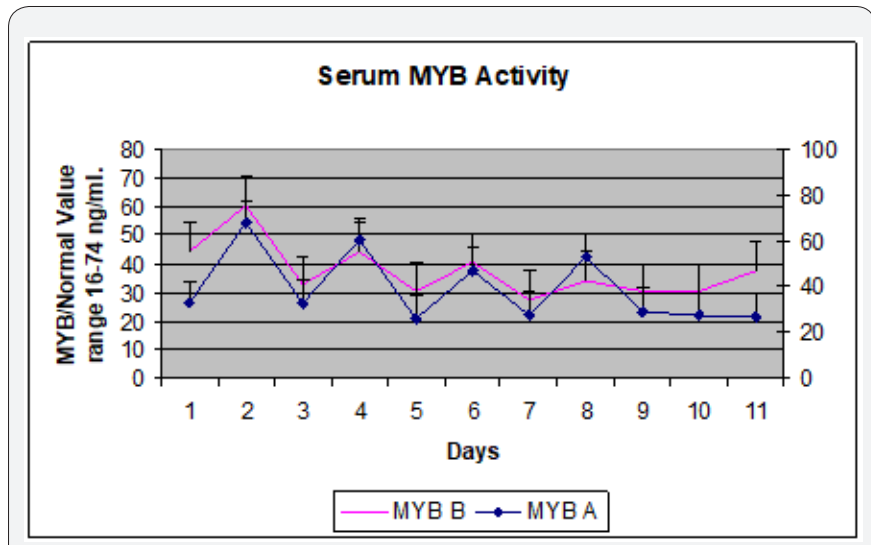

Figure 1: MYB.

*1: Pre-1st contest,2: Post-1st Contest,3: Pre-2nd contest,4: Post-2nd Contest,5: Pre-3rd contest,6:Post-3rd Contest,7: Pre-4th contest,8: Post-4th Contest, 9 : hours following the tournament, 10: hours following the tournament,11: hours following the tournament.

Creatine kinase and Lactate dehydrogenase was measured using the method of Autoanalyzer Spectrophotometer with a device of Japanese brand Olympus AU 2700.Myoglobin was measured using the method of Edia (Electroc hemilumine scenceimmu noassay) with a device of Japanese brand Elecsys 2010.Hemogram was measured using the method of Flow Sitometer with a device of USA brand Bekman Coulter LH 750 Analyzer. Centrifuge operations were conducted with a device of German brand Rotanta 460.Unit: CK-U/L(Unit/litre), LDH-U/L, MYB-NG/ML(Nanogram/ Millilitre)Descriptive statistics was applied to the basketball players in the study. Two-factor mixedmodel ANOVAs, with repeated measures on time were used and $\mathrm{t}$ test were applied so as to compare the biochemical values before and after the contest. In the analysis of the research data, Windows XP based SPSS 13 statistics program was used(Figures 1-3).

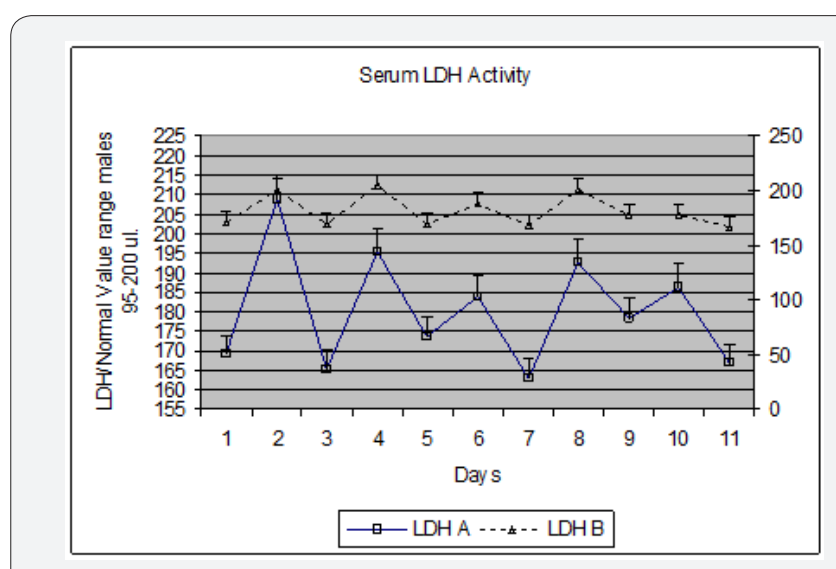

Figure 2: LDH.

*1: Pre-1st contest,2: Post-1st Contest,3: Pre-2nd contest,4: Post-2nd Contest,5: Pre-3rd contest,6:Post-3rd Contest,7: Pre-4th contest,8: Post-4th Contest,9: hours following the tournament, 10: hours following the tournament,11: hours following the tournament.

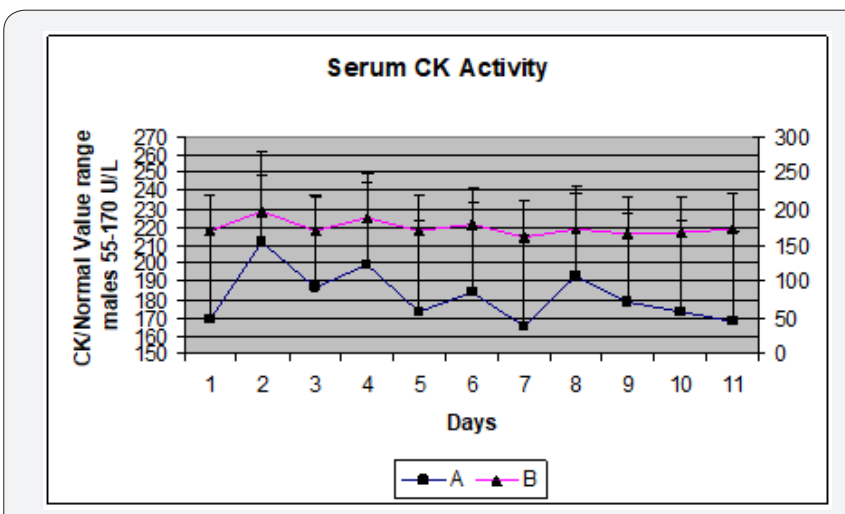

Figure 3: CK.

*1: Pre-1st contest,2: Post-1st Contest,3: Pre-2nd contest,4: Post-2nd Contest,5: Pre-3rd contest,6:Post-3rd Contest,7: Pre-4th contest,8: Post-4th Contest,9: hours following the tournament, 10: hours following the tournament,11: hours following the tournament.

\section{Results}

Statistical significance has been observed between contests and groups in terms of pre-contest and post-contest MYB values during the tournament $(\mathrm{p}<0,01)$. In recovery period, there isn't 
a statistically significant difference in the values measured 2448-72 hours later (Tables1-3).CK values were found statistically different in pre-contest and post-contest measurements. In recovery period, there hasn't been a significant difference between the groups in terms of the values measured 24-48-72 hours following the tournament. LDH values have been found statistically different in inter-contests, pre-contest and postcontest measurements $(p<0,01)$. In recovery period, a significant difference has been observed between the groups in LDH values measured 24-48-72 later $(\mathrm{p}<0,01)$ (Tables1-3).

Table 1: General features of the players.

\begin{tabular}{|c|c|c|c|c|c|c|c|c|c|}
\hline & & AGE(year) & Weight(Kg) & Height(Cm) & Sport Age(year) & Body Mass Index & Vertical Jump(Cm) & Flexibility (Cm) & P* \\
\hline G & N & M. Sd. & M. Sd. & M. Sd. & M. Sd. & M. Sd. & M. Sd. & M. Sd. & \\
\hline A & 10 & $23,7 \pm 2,7$ & $76,1 \pm 11,3$ & $181,3 \pm 8,1$ & $11,0 \pm 3,9$ & $23,0 \pm 3,9$ & $60,5 \pm 6,8$ & $20,9 \pm 8,2$ & $(p>0,05)$ \\
\hline B & 10 & $22,8 \pm 2,9$ & $69,9 \pm 7,6$ & $178,9 \pm 4,6$ & $8,2 \pm 3,5$ & $21,7 \pm 3,5$ & $55,6 \pm 6,5$ & $20,7 \pm 10,2$ & \\
\hline \multicolumn{7}{|c|}{$*:$ group comparison level of significance } & & & \\
\hline
\end{tabular}

Table 2: The markers at blood serum level of the players MYB, LDH, CK (Group A and B).

\begin{tabular}{|c|c|c|c|c|c|c|c|}
\hline \multirow[t]{2}{*}{ Day } & \multicolumn{2}{|c|}{ Study Timeline } & \multirow[t]{2}{*}{ Group } & \multirow[t]{2}{*}{$\mathbf{N}$} & \multirow{2}{*}{$\begin{array}{c}\text { Serum MYB activity } \\
\text { Mean-Std D }\end{array}$} & \multirow{2}{*}{$\begin{array}{c}\text { Serum LDH activity } \\
\text { Mean-Std D }\end{array}$} & \multirow{2}{*}{$\begin{array}{c}\text { Serum CK activity } \\
\text { Mean-Std D }\end{array}$} \\
\hline & & & & & & & \\
\hline \multirow[t]{4}{*}{1} & 1 & Pre-ex & A & 10 & $32,79 \pm 8,19$ & $169,10 \pm 14,40$ & $169,10 \pm 14,40$ \\
\hline & & & B & 10 & $44,39 \pm 18,75$ & $170,20 \pm 8,42$ & $170,00 \pm 6,83$ \\
\hline & 2 & Post-ex & A & 10 & $67,97 \pm 21,79$ & $208,90 \pm 25,87$ & $212,10 \pm 29,41$ \\
\hline & & & $\mathrm{B}$ & 10 & $60,38 \pm 34,11$ & $200,00 \pm 21,75$ & $197,30 \pm 55,33$ \\
\hline \multirow[t]{4}{*}{2} & 3 & Pre-ex & A & 10 & $33,06 \pm 9,54$ & $165,30 \pm 8,03$ & $187,00 \pm 67,21$ \\
\hline & & & B & 10 & $32,67 \pm 6,38$ & $169,40 \pm 11,48$ & $169,60 \pm 8,78$ \\
\hline & 4 & Post-ex & A & 10 & $60,46 \pm 23,15$ & $195,50 \pm 9,89$ & $199,60 \pm 17,78$ \\
\hline & & & B & 10 & $44,17 \pm 23,76$ & $204,80 \pm 16,17$ & $187,30 \pm 21,96$ \\
\hline \multirow[t]{2}{*}{3} & 5 & Pre-ex & A & 10 & $26,34 \pm 7,91$ & $173,50 \pm 20,01$ & $173,50 \pm 20,01$ \\
\hline & & & $\mathrm{B}$ & 10 & $30,53 \pm 6,80$ & $169,00 \pm 19,28$ & $169,40 \pm 14,04$ \\
\hline \multirow[t]{2}{*}{4} & 6 & Post-ex & A & 10 & $47,29 \pm 15,46$ & $183,90 \pm 18,89$ & $183,90 \pm 18,89$ \\
\hline & & & $\mathrm{B}$ & 10 & $40,72 \pm 19,87$ & $187,20 \pm 13,64$ & $178,50 \pm 13,72$ \\
\hline \multirow[t]{2}{*}{5} & 9 & $24 \mathrm{~h}$ & A & 10 & $29,31 \pm 6,43$ & $178,30 \pm 20,47$ & $178,30 \pm 20,47$ \\
\hline & & & B & 10 & $30,22 \pm 8,47$ & $177,40 \pm 9,58$ & $166,50 \pm 15,69$ \\
\hline \multirow[t]{2}{*}{6} & 10 & $48 \mathrm{~h}$ & A & 10 & $27,38 \pm 5,74$ & $186,40 \pm 13,70$ & $173,60 \pm 20,83$ \\
\hline & & & $\mathrm{B}$ & 10 & $30,23 \pm 7,49$ & $177,85 \pm 15,56$ & $168,10 \pm 22,58$ \\
\hline \multirow[t]{2}{*}{7} & 11 & $72 \mathrm{~h}$ & A & 10 & $27,12 \pm 6,07$ & $166,80 \pm 18,91$ & $168,30 \pm 18,28$ \\
\hline & & & B & 10 & $37,67 \pm 15,37$ & $166,80 \pm 16,05$ & $171,60 \pm 18,26$ \\
\hline$*: t$ & com & level of & icance & & & & \\
\hline
\end{tabular}

*1MÖ: Pre-1st contest, 1MS: Post-1st Contest, 2MÖ: Pre-2nd contest, 2MS: Post-2nd Contest, 3MÖ: Pre-3rd contest, 3MS: Post-3rd Contest, 4MÖ: Pre-4th contest, 4MS: Post-4th Contest, 24h: hours following the tournament, 48h: hours following the tournament, $72 \mathrm{~h}$ : hours following the tournament.

Table 3: The markers at blood serum level of the players MYB, LDH, CK (Group AB).

\begin{tabular}{|c|c|c|c|c|c|c|c|}
\hline Days & \multicolumn{2}{|c|}{ Study Timeline } & Group & N & Serum MYB activity & Serum LDH activity & Serum CK activity \\
\hline & & & & & Mean-Std D & Mean-Std D & Mean-Std D \\
\hline 1 & 1 & Pre-ex & A+B & 20 & $38,59 \pm 15,29$ & $169,65 \pm 11,49$ & $169,55 \pm 10,98$ \\
\hline & 2 & Post-ex & A+B & 20 & $64,17 \pm 28,13$ & $204,45 \pm 23,70$ & $204,70 \pm 43,79$ \\
\hline 2 & 3 & Pre-ex & A+B & 20 & $32,86 \pm 7,90$ & $167,35 \pm 9,87$ & $178,30 \pm 47,50$ \\
\hline & 4 & Post-ex & A+B & 20 & $52,31 \pm 24,31$ & $200,15 \pm 13,89$ & $193,45 \pm 20,44$ \\
\hline 3 & 5 & Pre-ex & A+B & 20 & $28,43 \pm 7,50$ & $171,25 \pm 19,26$ & $171,45 \pm 16,96$ \\
\hline & 6 & Post-ex & A+B & 20 & $44,00 \pm 17,65$ & $185,55 \pm 16,12$ & $181,20 \pm 16,30$ \\
\hline 4 & 7 & Pre-ex & A+B & 20 & $27,77 \pm 6,43$ & $165,60 \pm 21,75$ & $163,55 \pm 20,52$ \\
\hline
\end{tabular}


Journal of Physical Fitness, Medicine \& Treatment in Sports

\begin{tabular}{|c|c|c|c|c|c|c|c|}
\hline & 8 & Post-ex & $\mathrm{A}+\mathrm{B}$ & 20 & $43,63 \pm 19,39$ & $196,75 \pm 18,61$ & $182,45 \pm 26,72$ \\
\hline 5 & 9 & $24 \mathrm{~h}$ & $\mathrm{~A}+\mathrm{B}$ & 20 & $29,77 \pm 7,34$ & $173,60 \pm 20,83$ & $172,40 \pm 18,75$ \\
\hline 6 & 10 & $48 \mathrm{~h}$ & $A+B$ & 20 & $28,81 \pm 6,66$ & $180,00 \pm 18,38$ & $170,85 \pm 21,33$ \\
\hline 7 & 11 & $72 \mathrm{~h}$ & $A+B$ & 20 & $32,40 \pm 12,60$ & $166,80 \pm 17,07$ & $169,95 \pm 17,86$ \\
\hline \multicolumn{5}{|c|}{ *:t group comparison level of significance } & & & \\
\hline
\end{tabular}

A statistically significant difference has been recorded between the group applied 10-minute recovery protocol and passive recreation after contest $(p<0,01)$. Although MYB values of 'Group A' applied recovery protocol was higher during exercise, but very close to those of other group in recovery period. Additionally, on the other days, the first peak values couldn't be reached in players. As for MYB values measured 2448-72 hours later, they remained at lower rate compared to the other group (Tables 1-3).

\section{Discussion}

The homogeneity of the male basketball player groups in terms of age, height, weight and training age is of essential in respect to the analysis of the effects of the contest on biochemical parameters and the effects of the 10-minute recovery protocol after the contest which group A was applied through biochemical parameters.

In the studies in literature, skeleton muscle damage level is dependent upon the data obtained through the assessment of enzyme and blood values at serum blood level that reaches the extracellular level. The peak period of creatine kinase (CK) increased after exercise varies according to the type, volume and duration of exercise. A number of studies reveal that CK values reach the peak point after 1-5days after the exercise [6-11] have identified that CK values increase after long-term exercise and reach the peak point 24-48 hours later in their study. In a similar study by[1]. the same results have been identified[12]in their study with high density cycle ergometre have seen that serum CK values increase ten times soon after exercise and come close to the values before exercise 24 hours later.

Various results have been achieved in different researches. Indicating that $\mathrm{CK}$ values reach the peak point 2-4 days after exercise, Smith and Miles(2000) stated that CK values reach the peak point 3-4days after leg resistant exercise [13]in their study on football players conducted measurements 18-20, 42-44hours later and pointed out that serum $\mathrm{CK}$ values reach the peak point in 22-24 hours[14]conducted 10-minute high density basketball stimulation exercise and measured $\mathrm{CK}$ values soon after exercise, 5-10-15minutes after exercise, 24-48-72-96hours after exercise. They concluded that CK values increased soon after exercise but came to normal level at the end of the 4th day. In similar studies,[6-10]the increase in total CK values implies occurrence of muscle damage.In view of these results, it appears that basketball contests affect CK blood parameters of the player, which shows skeletal muscle damage in players occurs during contest.
In reference to the measurements after contest, a significant statistical difference in CK values wasn't observed between the control group and the group subjected to 10-minute recovery protocol $(\mathrm{P}>0,05)$.According to the data acquired, total $\mathrm{CK}$ values vary from subject to subject. As stated in literature, there is a judgement that CK values aren't affected by recovery protocols.

These results support the similar studies in the same fields. In many studies, $[9,15] \mathrm{LDH}, \mathrm{AST}$, CK are intracellular cytoplasmic enzymes that turn to functionally related extracellular after cell death. Therefore, their existence as extracellular are always associated with cell death and tissue destruction. Thereby, it is used as diagnosis indicator of tissue destruction in infection or brain damage following cerebrovascular diseases in myocardium infarction and chronic liver failure in medicine [12]implemented exercise protocol with 20 different eccentric contractions onto non-dominant hand flexor muscles; carried out measurements before exercise, soon after exercise and 24-48-72-96hours later exercise; and concluded that LDH values increased soon after the exercise, reached the peak point in 24-48 hours and turned to pre-exercise values in 96hours . In another study by [16]. Plyometric exercise protocol with high density eccentric contractions was applied and the values were measured soon after exercise and 24-48-72hours later. It was found that LDH values starting to increase soon after exercise rose by about $100 \% 72$ hours later and the difference between the values measured 24 hours and 48-72hours later were stated to be statistically significant.[14]in their study that examined weight and muscle pain relations through 10-minute high density basketball technical exercises have put forward that LDH release that increase soon after exercise come to pre-exercise level 4 days later. Literature also presents similar results of LDH values in exercise-induced muscle damage studies,[13]Cool-down consists of movements whose intensity gradually decreases so that blood circulation and various body functions can turn back to the condition before exercise.

Cool-down exercises can be done in two ways; active or passive recreations. Passive recreation includes resting, sitting, lying or sleeping $[3,17]$ have demonstrated that in comparison with passive recreation, 4-minute recovery protocol between repeated Wingate tests (28\% of MAX V02) enhance total work and the decline in capillary blood lactate of trained hockey players is more than that in sedentary $[17,18]$ stated that of the recovery periods they have applied to different kinds, the most effective method is recovery protocol period.

These results support the similar studies in the field. According to [19]studies that are consistent with our findings, 
it has been indicated that soon after muscle damage myoglobin (MYB) Concentration reaches the peak point before CK values. That the pre-contest MYB values are lower than half-time and post-contest values implies that muscle damage has notably increased. However, it is informed that MYB values of football players come to resting values 24-48-72hours after contest [12] in the exercise protocol with high density eccentric contractions carried out at cycle ergometer, have come to the conclusion that $53 \pm 22,1$ myoglobin values come to $554,5 \pm 25,4$ soon after exercise. Showing parallelism with our study, 49, $7 \pm 12$, 4 MYB values measured 24 hours after exercise turn to their preexercise level [20]carried out one-hour weight training exercise protocol including 50 subjects. The values were measured soon after exercise, during the following 7 days, 10th and 12th days. Similar results to those of our study were obtained. In literature, MYB measurements support our study as well.

The exercise protocols carried out in these studies are many repeated studies including high density muscle contractions. The results of our study could be assessed as an explanatory factor for the measurement values not being as high as those in similar studies, [21-23] have found the difference of fall rate of muscle damage indicators between the group applied stretching is than the other group recovery protocols. Besides, recovery times in various lengths were reported in the studies analyzing energy outcomes, lactic acid values and active and passive recovery processes. Within these studies, it is revealed that the groups put to active recovery experience a more rapid recovery process compared to the other groups [24].According to these results, basketball contests affect MYB blood parameters of the player. MYB values at blood serum level measure before and after contest refer to a considerable difference between the groups $(p<0,05)$ the literature studies on the adaptation process of the trained players to the training support our findings. Increasing MYB values in blood during exercise correspondent with the relation of recovery process with loading intensity, muscle contractions, the energy consumed, oxygen carriage required at cellular level and oxygen gap were found to be significantly different from the values of the subjects put to 10-minute recovery protocol after exercise. No important difference was recorded between the groups in terms of the values measured 24-48-72 hours after exercise during the recovery period, which means that the results are similar in both of the groups $(p>0,05)$. In literature these studies there is evidence that MYB values start to rise soon after exercise and reach the peak level in the first 1-3hours. Moreover, MYB values at blood serum level measured 24hours after exercise came close to pre-exercise level in bothof the groups. In the group applied 10-minute recovery protocol, recovery values were found statistically significant $(\mathrm{p}<0,05)$ $[25,26]$ concluded that passive recreation results in slowly progressing recovery values and recovery protocol results in more rapid recovery curve.

Our study puts forward that MYB values that increase at the beginning of exercise come to pre-exercise level 24 hours after exercise. Although MYB values of Group A were found to be higher than those of Group B, in respect to recovery speed, it can be seen that a more rapid recovery takes place in the group applied recovery protocol.

\section{Conclusion}

Consequently, recent studies demonstrate that exercise causes muscle damage at micro level and the type and size of exercise is determinant in the amount of damage. Muscle damage is closely associated with race, gender, age and training condition. It has been revealed that the degree of damage varies from person to person, sometimes high sometimes low amount of damage, depend upon training level of the players and sporting experiences. It has also been indicated that muscle damage responses of players at blood serum level are at lower value and damage responses come to normal level within shorter time.

In respect to the present study, it can be said that there are muscle damage responses in the skeletal muscles of the basketball players at the end of the 4-day basketball tournament and these responses come to normal levels 24-48-72hours later. Besides, it has been revealed that muscle damage responses applied 10-minute recovery protocol after contest reach normal values more rapidly in comparison with the other group. As a result of this study it has been observed that the difference of LDH blood parameter is significant. In accordance with these results, basketball contests are influential on the LDH blood parameters of the player. LDH measurements indicate that the difference in recovery time is assessed as significant between the group applied 10-minute recovery protocol after contest and the one applied passive recreation.

\section{References}

1. Noakes TD (1987)Effect of Exercise on Serum Enzyme Activities in Humans. Sports Medicine 4(4):245-267.

2. Vaile JM, GillND, Blazevich AJ (2007) The Effect of Contrast Water Therapy on Symptoms of Delayed Onset Muscle Soreness. Journal of Strength \& Conditioning Research 21(3):697-702.

3. Günay and M ve Yüce A (1996)Futbol Antrenmanının Bilimsel Temelleri. Ed T Çolakoğlu. Seren Ofset, Ankara.

4. White JA, Quinn G, Al-Dawalibi M,Mulhall J (1982)Seasonal changes in cyclist's performance: The British Olympic Track Squad. British Journal of Sports Medicine 16(1):4-12

5. Lieber RL,Fridén J (2002)Morphologic and Mechanical Basis of Delayed-Onset Muscle Soreness. J Am Acad Ortho Surg 10(1): 67-73.

6. Clarkson PM, Byrnes WC, Mccormick KM, Turcotte LP,White JS (1986) Muscle Soreness and Serum Creatine Kinase Activity Following Isometric, Eccentric, and Concentric Exercise. Int J Sports Med 3:152151.

7. Clarkson PM, Kearns AK, Rourzier P, Rubin R,Thompson PD (2006) Serum Creaitine Kinaz Levels and Renal Function Measures in Exertional Muscle Damage. Medicine and Science in Sports and Exercise 38(4):623-627.

8. Clarkson PM, NosakaK,BraunB(1992)Muscle Function after ExerciseInduced Muscle Damage and Rapid AdaptationMedicine and Science in Sports and Exercise24(5):512-520. 
9. Clarkson PM,TremblayI(1998)Exercise-Induced Muscle Damage, Repair and Adaptation in Humans. JApplPhysiol65(1):1-6.

10. ClarksonPM,HubalMJ(2002)ExerciseInduced Muscle damage in Humans. Am J Phys Med Rehabil 81:52-69.

11. StaronSR, HikitaS(2000)Muscular Responses To Exercise And Training. Exercise And Sport Science, USA, pp.163-173.

12. JulieA, PlezbertDD, Jeanmarie R, BurkeP(2005)Effects of The Homeopathic Remedy Arnica Onattenuating Symptoms of Exerciseİnduced Muscle SorenessJ Chiropr MedFall4(3):152-161.

13. KraemerWJ, SpieringBA, VolekJS, MartinGJ, HowardRL, etal. (2009) Recovery from a National Collegiate Athletic Association Division I Football Game: Muscle Damage and Hormonal Status. Journal of Strength and Conditioning Research 23(1):2-10.

14. KostopoulosN, FatourosIG, Siatitsas I, BaltopoulosP, KambasA, JamurtzasAZ etal. (2004)Intense basketball-simulated exercise induces muscle damage in men with elevated anterior compartment pressure. Journal of Strength and Conditioning Research 118(3): 451458

15. GutteridgeJ(1995)Lipid Peroxidation and Antioxidants as Biomarkers of Tissue Damage. Clinical Chemistry41(12):1819-1828.

16. AlexandreEE, RodrigoLDR, PierreAS, Andre NM, Rafael P,et al.(2009) Laboratoryof Physiology and Biokinetics: Drop Jump and Muscle Damage Markers. Serbian Journal of Sports Sciences 3(2):81-84.

17. SpiererD, GoldsmithR, BaranD, HryniewiczK,KatzS (2004)Effects of Active vs Passive Recovery On Work Performed During Serial Supramaximal Exercise Tests .Int J Sports Med 25(2): 109-114.

18. DoddS,PowersS, Callender T,BrooksE (1984)Blood Lactate Disappearance at Various Intensities of Recovery ExerciseJApplPhsysiol 57(5):1462-1465.

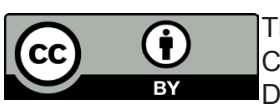

This work is licensed under Creative

Commons Attribution 4.0 License

DOI: 10.19080/JPFMTS.2018.05.555659
19. MikkelsenTS, ToftP(2005)Prognostic Value, Kinetics and Effect of Cvvhdf on Serum of the Myoglobin and Creatine Kinase in Critically Ill Patients with Rhabdomyolysis.Acta Anaesthesiologica Scandinavica49(6):859-864.

20. JonasP, Ulf H, PaulaP, ThomasB, UlfM, etal.(2007)Muscular Exercise Can Cause Highly Pathological Liver Function Tests in Healthy Men. British Journal of Clinical Pharmacology 65(2):253-259.

21. Ferri A, Narici M, Grassi B, Pousson M(2006)Neuromuscular Recovery after a Strength Training Session in Elderly People. Eur J Appl Physiol 97(3):272-279.

22.JamurtasA, TheocharisV, TofasT, TsiokanosA,YfantiC, etal.(2005) Comparison between Leg and Arm Eccentric Exercises of the Same Relative Intensity on Indices of Muscle DamageEur J Appl Physiol95(2-3):179-85.

23. JassalD, MoffatD, KrahnJ, AhmadieR, FangT, etal. (2009)Cardiac Injury Markers in Non-Elite Marathon Runners. Int J Sports Med 30(2):75-79.

24. DeclanAJ, ConnollyF, KevinMB, ChristieDL(2003)Effects Of Active Versus Passive Recovery on Power Output During Repeated Bouts of Short Term, High Intensity Exercise. Journal of Sports Science and Medicine 2(2): 47-51.

25. YoshidaT, WatariH,TagawaK(1996)Effects of Active And Passive Recoveries on Splitting of The Inorganic Phosphate Peak Determined by 31p-Nuclear Magnetic Resonance SpectroscopyNm Biomed 9(1):13-9.

26. SmithLL(1991)Acute inflammation: The underlying mechanism in delayed onset muscle soreness. Med Sci Sports Exerc 23(5): 542-551.

\section{Your next submission with Juniper Publishers} will reach you the below assets

- Quality Editorial service

- Swift Peer Review

- Reprints availability

- E-prints Service

- Manuscript Podcast for convenient understanding

- Global attainment for your research

- Manuscript accessibility in different formats ( Pdf, E-pub, Full Text, Audio)

- Unceasing customer service

Track the below URL for one-step submission https://juniperpublishers.com/online-submission.php 